\title{
INDEX OF SPECIES
}

Page numbers in italics refer to the main subjects of the papers

Acacia cyclops, 180

Aegilops, 390 speltoides, 329-339, 390

Agropyrum distichum, 180

Allium, 112, 372

cepa, 371

Amphibia, 11, 21-23

Anthophora urbana, 226

Anthoxanthum, 337

Antirrhinum majus, 391

Arabidopsis, 99

Arctotheca populifolia, 180

Artemia, 12

Ascobolus immersus, 397

Aspergillus, 119, 385

nidulans, 119, $120,121,125,128,129,131$, 263-284

Barley varieties, 341-357

Brassica, 394

campestris, 256

napus, 253-257

oleracea, 256

Carpobrotus edulis, 180

Cepaea hortensis, 188, 219

nemoralis, 177, 186, 187, 188, 213-221

sylvatica, 219

vindobonensis, 219

Charlock, see Sinapis arvensis

Chlamydomonas reinhardi, 394

Chrysochraon dispar, 218

Chorthippus brunneus, 259-262

Cisseps fulvicollis, 312

Cnidum subfruticosum, 180

Coenonympha tullia, 312

Coprinus, 125, 191, 192, 198, 397

lagopus, 125,126

Coregonus laveratus, 11

Crepis spp., 367-373

capillaris, $367-373$

fuliginosa, 367-373

neglecta, 367-373

Cyprinids, 11

Dactylis, 337 glomerata, 308

Dreissensia polymorpha, 127

Drosophila, 112

melanogaster, 95-100, 115, 128, 285-292
Enchytraeids, 12

Escherichia coli, 122, 124, 203-211, 395, 396

Euonymus europaeus, 230

Euptychia hermes, 308

Festuca, 337

Flax, var. Stormont Citrus, 71-81

Fragaria, 359

orientalis, 360

Galeopsis tetrahit, 324

pubescens, 324

speciosa, 324

Gasterosteus aculeatus, 11, 21-23

Gossypium barbadense, 324

hirsutum, 324

Helichrysum crassifolium, 180

Helix, 214, 215

Land snail, $177-190$

Lepus europaeus Pallas, 307

Linseed, varieties, 72

Loach, 11

Lolium perenne, 172, 375-379, 389

Lotus spp., 133

Lupinus nanus, 223-236

Lycaena dispar, 307

Maniola, 115

jurtina, 25-36, 308

Mouse, 91-93, 127, 307

Mus musculus, 307

Myzus persicae, 128

Nereis spp., 12

Neurosphora, 112, 121, 122

crassa, 119

Nicotiana rustica, 51-70, 99, 151-175, 269

Papaver spp., 37

dubium, 135-149, 152

Papilio machaon, 307

Pararge megera, 307-313

Pelargonium, 392

Phaseolus lunatus, 231

Phlox, 230

Phyciodes tharos, 312 
Pieris rapae, 307

brassicae, 307

Platichthys flesus, 11-24

Platypoecilus maculatus, 22

pleuronectids, 11

Pleuronectes platessa, 11-24

Poa timoleontis, 337

Pooideae, 337

Potentilla anglica, 315-327

erecta, 315-327

fruticosa, 359-362

reptans, 315-326

$\times$ mixta, 325, 326

Primula kewensis, 324, 326

foribunda, 326

verticillata, 326

vulgaris, 234

Proteus mirabilis, 123

Psathyrella, 397

Pulmonaria augustifolia, 230

Raspberry, 83-90

Saccharomyces cerevisiae, 117, 118, 395

Salmonella, 112

Salmonids, 11

Salmo salar, 11

trutta, 11
Satyrid butterfly, see Pararge megera

Schizophyllum commune, 126, 191-201

Sciurus vulgaris, 307

Secale, 337

Sinapis arvensis, 37-49

Sordaria, 397

Sorghum purpuro-sericeum, 337

Stickleback, see Gasterosteus aculeatus

Streptomyces coelicolor, 124

sturgeon, 11

Tetragonia decumbens, 180-183

Theba pisana, 177-190

Tilapia mossambica, 22

Tormentillae, 316

Tradescantia, 112

Tribolium, 101

castaneum, 247-252

Trifolium repens, 133, 392

subterraneum, 37

triticum aestivum, 390

speltoides, 329-337

turgidum, 329

Vicia, 112

faba, 368, 370

Xenopus laevis, 368, 372 